\title{
REMOVAL OF LENS FRAGMENTS FROM THE VITREOUS CAVITY
}

\author{
D. WONG, M. C. BRIGGS, M. U. HICKEY-DWYER and J. N. McGALLIARD \\ Liverpool
}

\begin{abstract}
SUMMARY
Between March 1993 and September 1994 we treated 25 cases of lenses in the vitreous cavity. Nineteen of the 25 were the result of dislocation during phacoemulsification. During this time, we adopted a single surgical algorithm involving vitrectomy, heavy liquids and ultrasound fragmentation. The aims of this retrospective study were to test the validity of our surgical algorithm and to report on outcomes and complications. The indications for vitreous surgery were raised intraocular pressure, uveitis and poor vision. Vitreous surgery was carried out at a mean of 29 days following phacoemulsification. Six patients required heavy liquids and 5 needed ultrasound fragmentation. Vitreous surgery undertaken less than 17 days after phacoemulsification had an increased likelihood of requiring heavy liquids and/or fragmentation $(p<0.02)$. The greatest threat to a favourable visual outcome was retinal detachment, which was significantly associated with fragmentation and use of heavy liquids $(p<0.02)$. The presence of an intraocular lens (IOL) reduced the surgical options for removal of the lens fragments, and IOL should not be inserted where lens matter dislocates. The study suggests that we should avoid fragmentation and, provided the intraocular pressure and uveitis can be controlled, that vitreous surgery should be deferred for 2-3 weeks following phacoemulsification.
\end{abstract}

There have been previous reports on the removal of lens fragments from the vitreous cavity using vitrectomy, ${ }^{1-5}$ with sodium hyaluronate, ${ }^{6}$ with heavy liquids ${ }^{7-9}$ and ultrasound fragmentation. ${ }^{10}$ In the earlier literature, the vitrectomies were performed via a single port using a multifunction vitreous cutting probe which incorporated infusion and illumination. ${ }^{1,4}$ Some of these cases involved whole lenses, subluxed or dislocated into the vitreous

Correspondence to: Mr David Wong, St Paul's Eye Unit, Royal Liverpool University Hospital, Prescot Street, Liverpool L7 8XP, UK. cavity spontaneously ${ }^{4,6}$ or following ocular trauma. ${ }^{4}$ These lenses were often mobile within the vitreous cavity. The other cases involved lens or lens fragments dislocated into the vitreous as a complication of cataract surgery. ${ }^{1-3,7,10}$ Needles were used to stabilise the lenses in order for the vitreous cutter to act upon them. ${ }^{4}$ Hard lenses presented another problem in that they could not always be removed using the vitreous cutter. ${ }^{4}$ Some needed to be delivered into the anterior chamber and removed via limbal or corneal incisions. ${ }^{4}$ The introduction of heavy liquids has enabled the surgeon to float the lens into the anterior segment for delivery. The use of ultrasound fragmentation in the vitreous cavity has been advocated by some authors. ${ }^{10}$ It has the advantage of emulsifying the lens, enabling it to be removed by aspiration and obviating the need to create a large anterior segment incision.

Posterior dislocation of lens fragments is a rare complication of phacoemulsification. ${ }^{11}$ The visual prognosis can be good but there is a high incidence of glaucoma, uveitis and retinal detachment. ${ }^{10,12}$ Previous reports have involved a relatively small number of cases and the best management for patients remains uncertain. In particular the following questions are pertinent:

Can small fragments be left?

What are the indications for vitreous surgery?

How urgent is it to remove the dislocated lens fragments following phacoemulsification?

Is it necessary to use heavy liquids?

What is the role of ultrasound fragmentation in the vitreous cavity?

What are the complications and how can they be prevented?

We conducted a retrospective review to address some of these questions. 


\section{PATIENTS AND METHODS}

Our unit provides a regional service, accepting patients as tertiary referrals from several hospitals in Merseyside. We reviewed the case records of all vitrectomy procedures undertaken for removal of lens from the posterior segment during the period March 1993 to September 1994. We treated 25 cases with lenses or lens fragments in the vitreous cavity. Four cases were dislocated intraocular lenses; 2 of the 4 patients presented with retinal detachments. There were 3 further patients with Marfan's syndrome and spontaneous dislocation of the crystalline lens (in 1 of these patients there was retinal detachment). For the purposes of this report these 6 patients were excluded.

The remaining 19 patients underwent vitrectomy for removal of crystalline lens fragments dislocated into the vitreous as 'a complication of phacoemulsification cataract surgery. There were 12 male and 7 female patients; the mean age was 64 years (range 42-89 years). Despite posterior capsule rupture 10 patients had apparently stable posterior chamber intraocular lenses; 1 had an anterior chamber lens implanted at the time of phacoemulsification. Eighteen of the 19 procedures were performed by Consultant surgeons and the remaining one by an Associate Specialist. Patients who had nuclear loss at the time of phacoemulsification but did not require vitreous surgery were not included in this study.

All patients had a large piece of lens material in the vitreous cavity, i.e. at least one-quarter of the nucleus. The indications for vitrectomy were raised intraocular pressure, uveitis and poor vision (Table I).

\section{Glaucoma}

Two patients had a history of glaucoma prior to cataract surgery. One had been successfully treated with peripheral iridectomy 12 months previously. The other patient was treated with combined phacoemulsification and trabeculectomy. The mean intraocular pressure was $29 \mathrm{mmHg}$ (range $16-60 \mathrm{mmHg}$ ) at the first visit to the vitreoretinal unit. Twelve of the 19 patients were being treated for raised intraocular pressure at the time of referral. Eight patients were on acetazolamide at the time of referral. The pressure continued to rise in 4 patients whilst awaiting vitreous surgery. One patient needed intravenous mannitol to bring the intraocular pressure down from a peak of $65 \mathrm{mmHg}$. Another patient received oral glycerol after intravenous acetazolamide failed to lower an intraocular pressure of $63 \mathrm{mmHg}$. High pressure prompted emergency surgery in 2 patients.

\section{Uveitis}

All 19 patients presented with anterior uveitis and varying degrees of vitritis. Two patients were being treated with systemic steroid for panuveitis. One patient had a hypopyon and an opaque vitreous when first examined by us 4 weeks after phacoemulsification. At the time of surgery 1 patient was found

Table I. Details of pre-operative findings, surgical management and post-operative condition of study patients

\begin{tabular}{|c|c|c|c|c|c|c|c|c|c|c|c|}
\hline \multirow[b]{2}{*}{ Patient } & \multicolumn{5}{|c|}{ Pre-operative } & \multicolumn{3}{|c|}{ Vitreous surgery } & \multicolumn{3}{|c|}{ Post-operative } \\
\hline & $\mathrm{VA}$ & IOP & Cornea & Uveitis & IOL & Procedure & Interval & Secondary IOL & IOP & VA & Complications \\
\hline 1 & $\mathrm{HM}$ & 14 & Oedema + & +++ & $\mathrm{PC}$ & Vitrectomy & 35 & - & 12 & $6 / 9$ & \\
\hline 2 & $\mathrm{CF}$ & 20 & Oedema + & ++ & - & Vitrectomy & 14 & $\mathrm{AC}$ & 8 & $6 / 12$ & \\
\hline 3 & $6 / 60$ & 24 & Clear & + & $\mathrm{PC}$ & Vitrectomy & 42 & - & 18 & $6 / 9$ & \\
\hline 4 & $\mathrm{CF}$ & 30 & Clear & + & - & Vit + PFL +Frag & 11 & Aphakic & 18 & $6 / 12$ & Retinal tear \\
\hline 5 & $\mathrm{HM}$ & 50 & Oedema & +++ & - & Vit + PFL + Section & 15 & $\mathrm{PC}$ & 16 & $6 / 9$ & \\
\hline 7 & $6 / 18$ & 40 & Oedema+ & + & PC & Vit + PFL + Frag & 192 & $\mathrm{AC}$ & 21 & HM & $\mathrm{RD}$ \\
\hline 8 & $6 / 60$ & 50 & Clear & ++ & - & Vitrectomy & 50 & $\mathrm{AC}$ & 21 & $6 / 9$ & \\
\hline 9 & PL & 63 & Oedemat & Hypopyon & $\mathrm{PC}$ & Vitrectomy & 31 & - & 18 & $6 / 9$ & $\begin{array}{l}\text { Pilocarpine }+ \\
\text { timolol }\end{array}$ \\
\hline 10 & HM & 10 & DM folds & + & - & Vitrectomy & 5 & $\mathrm{AC}$ & 16 & $6 / 9$ & \\
\hline 11 & $6 / 36$ & 38 & Bullae & + & PC & Vitrectomy & 42 & - & 22 & $6 / 6$ & \\
\hline 12 & $\mathrm{HM}$ & 16 & Oedema + & + & - & Vitrectomy & 27 & $\mathrm{PC}$ & 20 & $6 / 12$ & \\
\hline 13 & HM & 44 & Clear & +++ & - & Vit + PFL + Section & 2 & Aphakic & 20 & $6 / 18$ & RD \\
\hline 16 & PL & 23 & Oedema +++ & ++ & PC & Vit + PFL + Frag & 3 & - & 18 & NPL & RD \\
\hline 17 & $6 / 60$ & 23 & Clear & + & PC & Vit + PFL + Frag + Section & 15 & - & $?$ & $?$ & GRT \\
\hline 18 & $6 / 36$ & 44 & Oedema ++ & ++ & - & Vitrectomy & 21 & Aphakic & 20 & $6 / 60$ & $\mathrm{RD}$ \\
\hline 19 & $\mathrm{HM}$ & 40 & Oedema ++ & ++ & $\mathrm{PC}$ & Vitrectomy & 20 & - & 24 & $6 / 9$ & $\begin{array}{l}\text { Hyphaema, pupil } \\
\text { capture, timolol }\end{array}$ \\
\hline
\end{tabular}

Abbreviations: HM, hand movements; CF, counting fingers; (N)PL, (no) perception of light; DM folds, folds in Descemet's membrane; PC, posterior chamber lens implant; AC, anterior chamber lens implant; Vit, vitrectomy; PFL, perfluorocarbon liquids; Frag, ultrasound fragmentation; Section, ab externo section for delivery of the lens fragments; Secondary IOL, secondary intraocular lens inserted at vitrectomy; RD, retinal detachment; GRT, giant retinal tear.

Key: Interval refers to the time lapse between phacoemulsification and vitreous surgery. Pre-operative IOP and VA are the intraocular pressure $(\mathrm{mmHg})$ and visual acuity at the first assessment by the vitreoretinal unit. Post-operative IOP and VA are those recorded 6 months after vitreous surgery. 
to have a haemorrhagic retina with large cotton wool spots involving the posterior pole. This retinal appearance was presumed to be an inflammatory response and settled well post-operatively.

\section{Visual Acuity}

The mean visual acuity pre-operatively was less than $6 / 60$. One patient had a visual acuity at presentation of $6 / 18$ and an intraocular pressure of $40 \mathrm{mmHg}$. Another had a vision of 6/12 and a peak intraocular pressure of $28 \mathrm{mmHg}$ on acetazolamide. Fourteen patients had visual acuity of counting fingers or worse. The causes of the poor vision were corneal oedema, folds in Descemet's membrane and vitreous opacity.

\section{Retinal Detachment}

One patient had a small peripheral retinal detachment arising from atrophic retinal holes in the inferotemporal quadrant. The detachment was only found at the time of surgery and it was uncertain whether it was pre-existing and unrelated to the phacoemulsification. One had a previous perforating injury and this gave rise to the cataract formation. No view of the retina was available prior to his cataract surgery. He subsequently went on to develop a retinal detachment following phacoemulsification and vitrectomy.

\section{Timing of Surgery}

From the outset we made a deliberate decision to regard the uncomplicated patients as urgent but not emergencies. Patients with dislocated lens fragments were treated with the same priority as macula-off retinal detachments and were offered treatment at the next available list. The timing of surgery was therefore determined partly by our workload but also by the pattern of referral. Some surgeons were

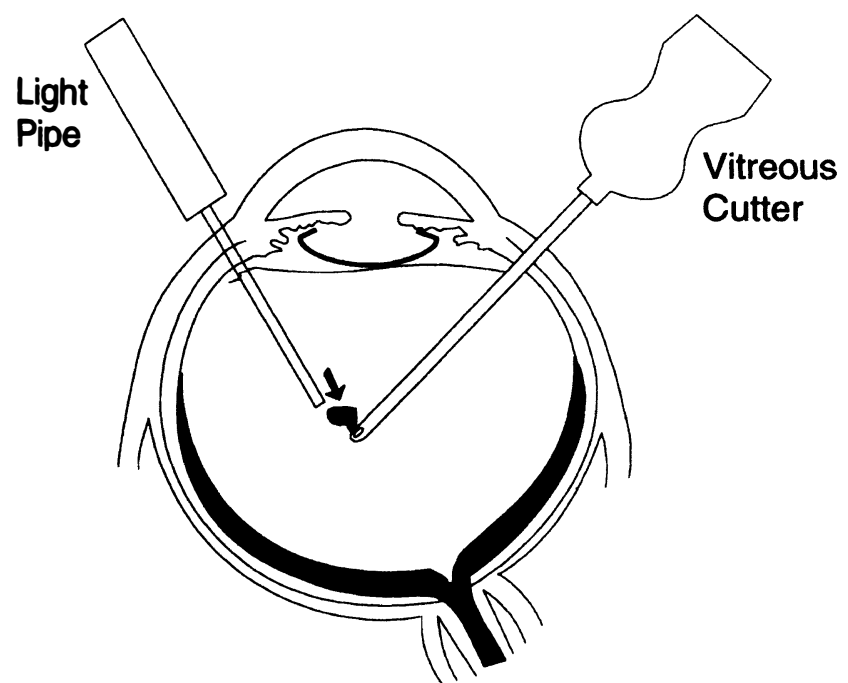

Fig. 1. 'Force feeding' of lens material into the vitreous cutter. very keen for patients to be assessed by the vitreoretinal team immediately after phacoemulsification. Other surgeons tried a period of conservative treatment to see whether the lens fragments might absorb spontaneously without vitreous surgery. Urgent surgery was sometimes required for some of these cases when conservative measures failed, as by the time they were referred they had high intraocular pressure or severe uveitis unresponsive to medical treatment. These patients received emergency vitrectomies. The mean interval between phacoemulsification and referral to the vitreoretinal unit was 27 days. The mean interval between phacoemulsification and vitrectomy was 29 days. The average interval was skewed by 1 patient who was not referred until 5 months after cataract surgery. Despite having a sizeable lens fragment in the vitreous cavity, he enjoyed a vision of $6 / 18$ after cataract surgery. At 5 months, he developed raised intraocular pressure $(40 \mathrm{mmHg})$ and corneal oedema which prompted his referral to us.

\section{Surgical Algorithm}

All patients were treated with pars plana vitrectomy. When the lens material was soft, it was removed by the vitreous cutter. We employed a bimanual technique of using the light pipe to 'force feed' lens material into the vitreous cutter. The lens fragments were engaged by vitreous cutter using aspiration. We then used the light pipe to push the lens fragments into the port before activating the guillotine action of the vitreous cutter (Fig. 1). In aphakic patients there was usually lens matter in the anterior chamber. The lens material aggregated in the inferior part of the anterior chamber and was often adherent to the endothelium, the angle and the iris. This was removed by aspiration. Because of the expense, we only used perfluoro- $n$-octane liquid (PFL) after we had attempted and failed to remove the lens material

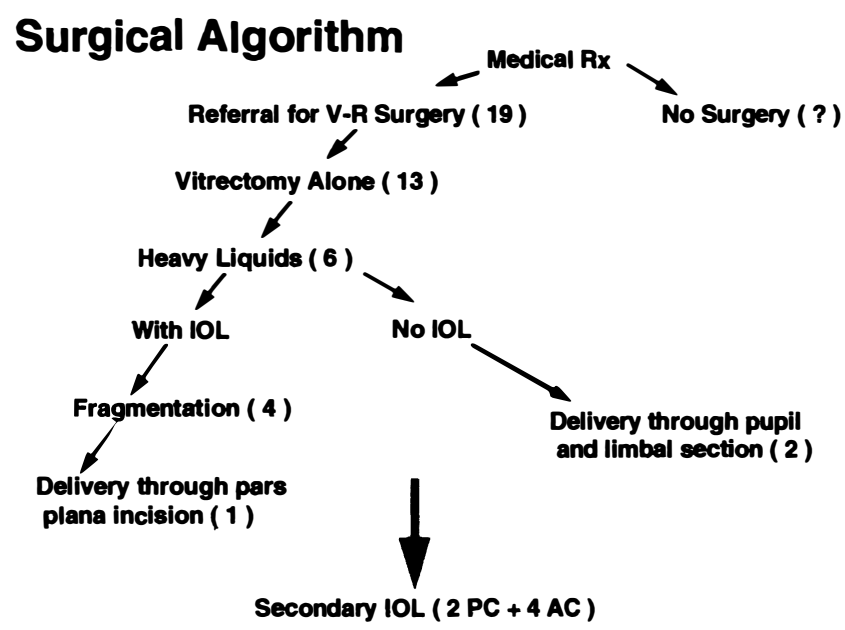

Fig. 2. Surgical algorithm. Numbers in parentheses indicate the number of patients undergoing each procedure. 
using the vitreous cutter. For the hard lens fragments that were 'indigestible', we used PFL to float the lens fragments into the anterior segment. In aphakic patients, lenses were delivered through the pupil and via a limbal incision. In pseudophakic patients, the exit route through the pupil was blocked. We then employed ultrasound fragmentation. A small amount of PFL was used to lift the lens material from the retina and fragmentation was applied keeping a relatively safe distance from the retina. When the lens fragments were so hard and mobile that even ultrasound failed, as a last resort a large pars plana incision would be made to deliver the lens fragments. The surgical algorithm is summarised in Fig. 2. With one exception (see below) we adhered to this algorithm.

Using this algorithm, we found that it was possible to remove the lens fragments using vitreous cutter alone in 13 of 19 patients. Six patients had hard lenses and needed PFL. Of the 6 patients, 3 were aphakic. We delivered the lenses through the pupil and via a limbal incision in 2 of 3 patients. In the remaining 1 aphakic patient we departed from the algorithm and applied ultrasound fragmentation rather than delivering the lens fragments via a limbal section. A total of 4 patients received fragmentation in the vitreous cavity. In 1 patient, the fragmentation was unsuccessful. The exit via the pupil was blocked by a posterior chamber lens implant. A $90^{\circ}$ circumferential incision in the pars plana was made temporally to deliver the hard lens material.

Secondary intraocular lens implants were inserted at the time of vitrectomy in 6 patients. There was sufficient posterior capsule to support a posterior

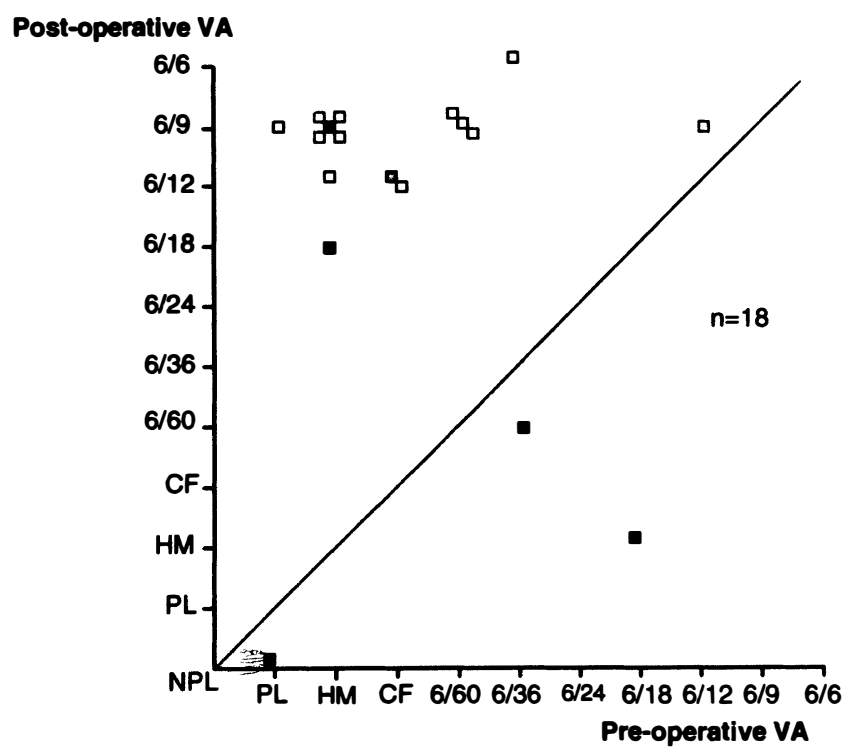

Fig. 3. Visual acuity scattergram: post-operative visual acuity plotted against pre-operative visual acuity. Open squares, no retinal complications; black squares, retinal detachment; grey square, retinal tear. chamber lens in 2 patients. The remaining 4 patients received anterior chamber lens implants. We elected not to attempt secondary intraocular lens implants in 4 patients because of associated complications: peroperative posterior retinal tears cause by fragmentation probe, previous perforating injury and corneal oedema.

\section{RESULTS}

Eighteen of the 19 patients completed a minimum 6 months of follow-up. The nineteenth patient was lost to follow-up within 2 months of vitrectomy.

\section{Glaucoma}

Intraocular pressure was satisfactory in 17 of the 18 patients post-operatively, in 1 patient satisfactory pressure was maintained by a topical beta-blocker, and 1 patient had an intraocular pressure of $24 \mathrm{mmHg}$ on no treatment.

\section{Uveitis}

Intraocular inflammation settled well in all 18 eyes. In the case with hypopyon Staphylococcus albus was cultured from the vitreous aspirate; the eye achieved $6 / 9$ vision.

\section{Visual Acuity}

All patients who had uncomplicated surgery achieved good vision post-operatively. Fourteen of the 18 patients achieved a best corrected visual acuity of $6 / 12$ or better (Fig. 3), 1 patient of $6 / 18,1$ of $6 / 60,1$ of hand movements (HM) and 1 of no perception of light (NPL). Poor vision was associated with retinal detachment (5 patients).

\section{Retinal Tears/Detachment}

The most significant complication was retinal detachment. The 1 patient in whom a pars plana incision was required to deliver a hard nucleus suffered a giant retinal tear. This woman was treated with silicone oil. She was subsequently lost to. follow-up. Two further patients had retinal breaks detected intraoperatively, which were treated by laser retinopexy; 1 patient subsequently suffered retinal detachment, the other no further complications. A total of 5 patients developed retinal detachments. Three retinas were successfully reattached, resulting in a visual acuity of $6 / 9,6 / 18$ and $6 / 60$, respectively. Of the 2 patients whose retinas failed to reattach 1 had a visual acuity of NPL and the other of HM at 6 months.

Table II. Retinal complications and surgical techniques

\begin{tabular}{lcc}
\hline & RD/retinal tear & No RD/retinal tear \\
\hline Vitrectomy & 2 & 11 \\
Vitrectomy plus & 5 & 1 \\
\hline
\end{tabular}


Table III. Retinal complications and interval between phacoemulsification and vitreous surgery

\begin{tabular}{lcc}
\hline & Interval $<17$ days & Interval $>17$ days \\
\hline Vitrectomy & 2 & 11 \\
Vitrectomy plus & 5 & 1 \\
\hline
\end{tabular}

\section{Analysis of Results}

We examined the incidence of retinal detachment or retinal tear as a complication of the surgical procedure. We divided the patients into two groups according to which surgical techniques were used. The first group comprised 13 patients who had removal of lens material by vitrectomy only. The second group included 6 patients who, in addition to vitrectomy, required other manoeuvres, i.e. use of heavy liquids, fragmentation or limbal/pars plana incision (vitrectomy plus). Table II shows these data. Analysis of the data by Fisher's exact test demonstrates a significant correlation between the use of surgical techniques in addition to vitrectomy and the occurrence of retinal complications $(p<0.02)$.

\section{Timing of Surgery}

We analysed the outcome for each patient in terms of the incidence of retinal detachment and the interval between phacoemulsification surgery and vitrectomy. These data are shown in Table III. There is a significant correlation between early vitrectomy (i.e. $<17$ days) and the incidence of use of techniques in addition to vitrectomy and the incidence of retinal complications $(p<0.02$, Fisher's exact test $)$.

\section{DISCUSSION}

Vitrectomy techniques were successful in treating raised intraocular pressure and uveitis and final visual acuity was good in the majority of cases. There was, however, an unacceptably high incidence of retinal detachment following the removal of lens fragments in our series. There was a close correlation with the use of techniques in addition to vitrectomy and subsequent retinal detachment. Poor visual outcome was due to retinal detachment.

The surgical technique used was determined by the nature of the lens fragments. Those soft enough were removed by vitreous cutter alone; those too hard for the vitrector were removed using heavy liquids and/ or fragmentation. Our analysis of the timing of surgery shows that those patients whose vitreous surgery is delayed ( $>17$ days) are less likely to require these additional techniques. The reason for this might be that the lens material will become hydrated and therefore softer, allowing removal using only the vitreous cutter. In 3 cases retinal tears were detected at the time of vitrectomy. One of these was caused by pars plana incision, the other 2 by the lens nucleus being pushed against the retina by the fragmatome.
The incidence of retinal detachment was far higher than that following extracapsular cataract extraction complicated by vitreous loss. ${ }^{13}$ The reasons for this may include the continued use of the phacoprobe after the posterior capsule was breached. Retinal tears caused at the time of cataract surgery might not give rise to retinal detachment until vitrectomy exposes the retinal tears to intraocular currents. ${ }^{14}$ There was a close correlation with the use of techniques in addition to vitrectomy (heavy liquids, fragmentation, pars plana incision) and subsequent retinal detachment. We think the reasons for this must be due largely to the use of the fragmatome in the vitreous gel. Both phacoprobe and fragmatome are designed to work in the lens. They do not have the ability to cut vitreous gel. Aspiration with these instruments without cutting might give rise to traction and retinal tear formation. Lambrou and Stewart ${ }^{10}$ described the use of fragmentation in the vitreous cavity. They reported retinal tears in 4 of 8 cases. The high incidence of retinal tears with fragmentation may be due to incomplete posterior detachment in these cases even after 'core vitrectomy'.

It is our strong recommendation that ultrasound probes should not be used in the vitreous cavity. When the posterior capsule is breached during cataract surgery the phacoemulsification probe should be withdrawn, the remaining lens matter delivered via an ab externo incision and any vitreous loss managed by a thorough anterior vitrectomy. Should fragments of nuclear material be dropped into the vitreous cavity we would strongly advise against the insertion of an intraocular lens (IOL). The presence of an IOL necessitated the use of fragmentation in 3 patients, all of whom developed retinal complications.

Most cataract surgeons thought it was reasonable to commence medical treatment initially - hence the mean interval to referral of 27 days. Based on our experience of treating our patients it is impossible to make any recommendations regarding the size of fragments that can safely be left. We do, however, advocate early referral to the vitreoretinal service as intraocular pressure may rise precipitously requiring urgent surgical treatment. The results of this series suggest that those patients who had delayed vitreous surgery had fewer complications and enjoyed better visual outcome.

\section{Conclusions}

Lens dislocation is a rare complication of phacoemulsification. Should it occur we recommend the following, based on our experience in this retrospective study:

Do not insert an intraocular lens.

Initial medical treatment. 
Make an early referral to the vitreoretinal service. Carry out early vitreous surgery if intraocular pressure or uveitis is uncontrolled, delivering the lens through a limbal or corneal section.

Delay surgery with medical treatment if the intraocular pressure is controlled, to allow hydration and removal of the lens using the vitreous cutter alone.

Key words: Phacoemulsification, Retinal detachment, Vitrectomy, Heavy liquids, Lens, Fragmentation .

\section{REFERENCES}

1. Michels RG, Shacklett Col DE. Vitrectomy technique for the removal of retained lens material. Arch Ophthalmol 1977;95:1767-73.

2. Hutton WL, Snyder WB, Vaiser A. Management of surgically dislocated intravitreal lens fragments by pars plana vitrectomy. Trans Am Acad Ophthalmol Otolaryngol 1978;85:176-89.

3. Fastenberg DM, Schwartz PL, Shakin JL, Golub BM. Management of dislocated nuclear fragments after phacoemulsification. Am J Ophthalmol 1991;112: 535-9.

4. Peyman GA, Raichand M, Goldberg MF, Ritacca D. Management of subluxated and dislocated lenses with the vitrophage. Br J Ophthalmol 1979;63:771-8.

5. Blodi BA, Flynn HW Jr, Blodi CF, Folk JC, Daily MJ. Retained nuclei after cataract surgery. Ophthalmology 1992;99:41-4.

6. Haymet BT. Removal of a dislocated hypermature lens from the posterior vitreous. Aust NZ J Ophthalmol 1990;18:103-6.

7. Rowson NJ, Bacon AS, Rosen PH. Perfluorocarbon heavy liquids in the management of posterior dislocation of the lens nucleus during phacoemulsification. $\mathrm{Br}$ J Ophthalmol 1992;76:169-70.

8. Lewis H, Blumenkranz MS, Chang S. Treatment of dislocated crystalline lens and retinal detachment with perfluorocarbon liquids. Retina 1992;12:299-304.

9. Wallace RT, McNamara JA, Brown G, Benson W, Belmont J, Goldberg R, Federman J. The use of perfluorophenanthrene in the removal of intravitreal lens fragments. Am J Ophthalmol 1993;116:196-200.

10. Lambrou FH Jr, Stewart MW. Management of dislocated lens fragments during phacoemulsification. Ophthalmology 1992;99:1260-2; discussion 1269-9.

11. Seward HC, Dalton R, Davis A. Phacoemulsification during the learning curve: risk/benefit analysis. Eye 1993;7:164-8.

12. Korobelnik JF, Nabet L, Frau E, Elmaleh C, Hanna K, Pouliquen Y. Use of perfluorocarbon solutions in the surgical treatment of posterior luxation of the lens. J Fr Ophtalmol 1992;15:235-42.

13. Acheson JF, McHugh JD, Falcon MG. Changing patterns of early complications in cataract surgery with new techniques: a surgical audit. Br J Ophthalmol 1988;72:481-4.

14. Machemer R. The importance of fluid absorption, traction, intraocular currents, and chorioretinal scars in the therapy of rhegmatogenous retinal detachments. XLI Edward Jackson memorial lecture. Am J Ophthalmol 1984;98:681-93. 